\title{
Sobrevivencia de pacientes diagnosticados con cáncer gástrico en el occidente de Honduras, periodo 2002-2012
}

\author{
Karla Patricia Torres Ortiz, Columba Rodríguez ${ }^{1}$ \\ Héctor Sandoval, Ana Julia Melgar ${ }^{2}$ \\ Pedro Sauceda ${ }^{3}$
}

\section{RESUMEN}

El presente trabajo de investigación describe la sobrevivencia a 5 años de los pacientes diagnosticados con cáncer gástrico del occidente del país, se determinaron los factores que afectan la tasa de sobrevida, el porcentaje de pacientes que no tuvieron tratamiento, por qué no lo recibieron, la sobrevivencia según el tratamiento recibido y si influyen o no los indicadores de pobreza en la sobrevivencia del paciente.

Para realizar la investigación se elaboraron dos tipos de instrumentos: la autopsia verbal, la cual permitió conocer la evolución de la enfermedad desde su diagnóstico hasta el momento del fallecimiento y el instrumento de los indicadores de pobreza, que reflejó la influencia directa de la calidad de vida en la evolución de la enfermedad.

El estudio es probabilístico, con un universo de 490 y una muestra de 154 casos, dentro de los resultados más relevantes se pueden describir que: la sobrevivencia a 5 años después del diagnóstico de cáncer gástrico en la zona occidental de Honduras es del $14.29 \%$, dato que resulta inferior con relación al $20 \%$ propuesto en la hipótesis.

De los 22 casos que representan la sobrevivencia, 15 son del sexo masculino $(9.74 \%)$ y 7 del sexo femenino, equivale a $4.55 \%$

\footnotetext{
${ }^{1}$ Beneficiarias de una beca básica de la DICYP, profesoras e investigadoras, Departamento de Química y Biología, Centro Universitario Regional de Occidente, (CUROC-UNAH): ktorres@unah.edu.hn

${ }^{2}$ Beneficiarios de una beca básica de la DICYP, profesores investigadores, Departamento de Enfermería, Centro Universitario Regional de Occidente, (CUROC-UNAH):

${ }^{3}$ Beneficiario de una beca básica de la DICYP, Jefe del área de informática y profesor, Centro Universitario Regional de Occidente, (CUROC-UNAH):
} 
Se concluye que la población de occidente es altamente susceptible a padecer esta patología debido a factores genéticos y ambientales, que la población en general no toma conciencia de cómo prevenir cualquier situación de salud y que a nivel de la secretaria de salud no existe un departamento que controle, regule y registre todos los casos de los diferentes tipos de cáncer y mucho menos sus estadios.

Palabras clave: cáncer gástrico, autopsia verbal, indicadores de pobreza, diagnóstico, sobrevida, tratamiento, patología estadio.

\section{ABSTRACT}

The present research describes the 5 year survival period for patients diagnosed with gastric cancer who live in the western part of Honduras. It determines the factors affecting survival rate, the percentage of non treated patients, reasons why they were not treated, and the survival period according to the type of treatment received. This study also indicates if whether or not poverty indicators have an influence on patient survival.

Two instruments were developed to conduct the research: 1) verbal autopsy, which allowed the evolution of the disease from diagnosis to the time of death and 2) the instrument of poverty indicators, reflecting the direct influence of the quality of life in the progression of the disease.

This probabilistic study analized 154 cases in a universe of 490 patients. The results show that only $14.29 \%$ of the patients have a 5 year survival period after gastric cancer diagnosis, which is lower than the $20 \%$ proposed in the hypothesis. 22 cases represented this 5 year survival period: 15 cases were male patients $(9.74 \%)$ and 7 cases were female patients (equivalent to $4.55 \%$ )

Due to genetic and environmental factors in the western part of Honduras, its population is highly susceptible to suffer from this disease. In addition, the general population has no idea of health care prevention and there is no state health departament control whatsover to regulate and record all types of cancer cases, much less its pathology.

Keywords: gastric cancer, verbal autopsy, poverty indicators, diagnosis, survival, treatment, pathology 


\section{INTRODUCCIÓN}

El presente estudio abre un sendero más en la investigación del cáncer gástrico, siendo este el primer estudio de sobrevivencia de cáncer gástrico en Honduras, ya que hasta el momento no se registra ningún estudio científico orientado a conocer la tasa de sobrevida, la frecuencia con que se recibe tratamiento para esta patología y los resultados de los mismos; asimismo, permite entrever los resultados de la política actual de seguimiento y establece la satisfacción de las necesidades de tratamiento de los pacientes con cáncer gástrico en la zona.

El estudio consistió en realizar una autopsia verbal con los familiares o las personas más cercanas de los pacientes diagnosticados y fallecidos por cáncer gástrico mediante una metodología que permitió reconstruir la historia y el camino recorrido por un paciente desde que se enfermó hasta que falleció.

El análisis de la información obtenida permitió identificar las poblaciones y factores de riesgo, así como fallas en los sistemas de salud, para que a nivel institucional se establezcan medidas a nivel local y departamental con estrategias que permitan mejorar los procesos de atención.

Los retos identificados para el estudio están relacionados principalmente con la información mal registrada, o parcialmente archivada, como datos incompletos, dirección y procedencia incompleta. Sin embargo, este inconveniente fue superado con el apoyo del Registro Nacional de las Personas, registros médicos del Hospital Regional y con la participación activa de los estudiantes que viven en las comunidades en estudio.

\section{MÉTODO}

El tipo de estudio utilizado en esta investigación es tipo no experimental, tomando como información la unidad de endoscopía del Hospital Regional de Occidente.

El diseño transeccional exploratorio permitió conocer el comportamiento de la enfermedad, realizando el cruce de los datos de las variables en los dos instrumentos utilizados (autopsia verbal e indicadores de pobreza) del que se obtuvo un panorama de la situación actual en la evolución de la enfermedad y sistemas de salud. 
El enfoque es cuantitativo y se siguió una secuencia de la evolución de la enfermedad desde el diagnóstico hasta la muerte del paciente; es probatorio, ya que se utilizaron datos fidedignos de los casos registrados de cáncer gástrico en el occidente de Honduras, comprendidos en los años 2002-2012.

Los datos se ingresaron a una base en Excel (Microsoft office 2013 para Windows 7) y posteriormente se realizó el análisis estadístico con SPSS 18; también se estimó la probabilidad de supervivencia con Kaplan Meier; se analizaron los factores que cambian la supervivencia mediante modelos de riesgos proporcionales de Cox., y se calcularon los diferentes riesgos de fallecer tras el diagnóstico de cáncer gástrico con un intervalo de confianza del 95\% usando los modelos de regresión.

La unidad de análisis de la investigación fueron pacientes, hombres o mujeres, mayores de 18 años que fueron atendidos en las salas de medicina de Hombres y mujeres y diagnosticados con cáncer gástrico mediante endoscopia y biopsia en el Hospital Regional de Occidente

\section{Universo y muestra}

Cuantitativo: 490 pacientes diagnosticados con cáncer gástrico en el Hospital Regional de Occidente en el período comprendido entre los años 2002-2012.

La muestra es tipo probabilística no estratificada, se tomaron grupos de población de la muestra en cada uno de los departamentos Copán, Ocotepeque y Lempira.

El universo fue de 490 casos, el cálculo de la muestra fue de 154 casos, según la fórmula de Murray y Larry (2005):

Temporalidad: es una investigación de tipo longitudinal retrospectivo, ya que se analizó la sobrevivencia de los pacientes diagnosticados con cáncer gástrico diez años atrás (2002-2012).

$$
\mathrm{n}=\frac{\mathrm{Z}_{\mathrm{a}}^{2} \cdot \mathrm{N} \cdot \mathrm{p} \cdot \mathrm{q}}{\mathrm{i}^{2}(\mathrm{~N}-1)+\mathrm{Z}_{\mathrm{a}}^{2} \cdot \mathrm{N} \cdot \mathrm{p} \cdot \mathrm{q}}
$$




\section{Intervenciones}

Se realizó la prueba piloto como trabajo preliminar, la cual permitió validar el instrumento de la autopsia verbal e indicadores de pobreza previa autorización por el entrevistado, cumpliendo con los lineamientos de trabajo ya establecidos para aplicarla. Como aplicadores de la prueba piloto se seleccionaron a 15 estudiantes de último año de la Carrera de Enfermería, por la vinculación con el tema de estudio, tomando como referencia para la asignación del trabajo de campo la zona donde viven y trabajan cada uno de ellos, en concordancia con la dirección de los pacientes.

Los estudiantes fueron capacitados por los investigadores para posteriormente ser supervisados al momento de realizar la recolección de la información en el mismo lugar y evitar márgenes de error.

Este grupo que lideró y supervisó el proceso de la entrevista, después de la aplicación de la prueba piloto dio a conocer los efectos del ensayo prueba-error y así quedar validado el instrumento. Además, fueron responsables con la ayuda de los investigadores de capacitar al resto de estudiantes de otras carreras del CUROC, que participaron en el levantamiento de la totalidad de las entrevistas.

Una vez validado el instrumento se procedió a capacitar al resto de estudiantes de las asignaturas de Biología y Química, los cuales se seleccionaron de la base de datos del registro docente (lista de alumnos matriculados), con un total de 71 capacitados. Después se seleccionaron de acuerdo a habilidades individuales y según la procedencia o lugar en donde reside cada uno; de esta manera se facilitó la ubicación de los pacientes de la base central de datos de cáncer de gástrico de los tres departamentos: Copán, Lempira y Ocotepeque.

\section{Análisis estadístico}

Se realizó el análisis de datos cuantitativos utilizando las medidas estadísticas de tendencia central con la frecuencia, porcentajes, media y otras. Para el análisis cuantitativo y aplicación del modelo de Cox se utilizó el programa computacional SPSS 18 como herramienta para realizar los cálculos necesarios y Ms Excel para realizar los gráficos para el análisis. Otro aspecto importante es que a lo largo del proceso de investigación para la obtención de los datos, se tomaron en cuenta tres aspectos: las personas, el contexto y el tiempo. 


\section{Regresión de Cox}

El análisis de la supervivencia se conoce como regresión de Cox y también como modelo de riesgos proporcionales, son usados para modelar los riesgos que afectan a la supervivencia de una población de sujetos (recibe su nombre del estadístico británico David Cox. Formulación:

El modelo de Cox expresa la función de riesgo instantáneo de muerte $\lambda$ en función del tiempo t y de variables $X_{1}, \cdots, X_{n}$ así:

$$
\lambda\left(t, X_{1}, \cdots, X_{n}\right)=\lambda_{0}(t) \exp \left(\Sigma_{i=1}^{n} \beta_{i} X_{i}\right)
$$

A $\lambda_{0}(t)$ se le denomina riesgo base y corresponde al riesgo de muerte cuando todas las variables tienen valor 0 (posiblemente, después de una reparametrización). Es la única parte de la expresión que depende del tiempo:

La otra: $\exp \left(\sum_{i=1}^{n} \beta_{i} X_{i}\right)$, solo depende del resto de las variables. (Boj del Val, pág. 3)

\section{La hipótesis de los riesgos proporcionales}

El modelo no busca tanto estimar la función: $\lambda_{0}(t)$, que es idéntica para todos los sujetos, como la relación entre los riesgos de muerte entre dos individuos expuestos a factores distintos. Para ello, el modelo parte de una hipótesis.

Fundamental: que los riesgos son proporcionales. Para comprender esta noción, puede estudiarse el caso de dos individuos, i y j que solo se diferencian en la k-ésima variable. Supóngase que vale 0 para i y 1 para j:

$$
\frac{\lambda(t, j)}{\lambda(t, i)}=\frac{\lambda_{0}(t) \exp \left(\beta_{1} X_{1}^{\prime}+\cdots+\beta_{k-1} X_{k-1}^{\prime}+\beta_{k} \times 1+\beta_{k+1} X_{k+1}^{\prime}+\beta_{n} X_{n}^{\prime}\right)}{\lambda_{0}(t) \exp \left(\beta_{1} X_{1}^{\prime}+\cdots+\beta_{k-1} X_{k-1}^{\prime}+\beta_{k} \times 0+\beta_{k+1} X_{k+1}^{t}+\beta_{n} X_{n}^{\prime}\right)}=\exp \left(\beta_{k}\right)
$$

Entonces, para cualquier tiempo t, la fórmula muestra que el cociente es independiente del tiempo.

Al utilizar la regresión de Cox es necesario verificar que se cumple dicha hipótesis. Para ello es necesario comprobar que el efecto de cada variable es constante en el tiempo. Existen varios métodos; puede utilizarse un método gráfico, si una variable, por ejemplo, toma únicamente los valores 0 y 1 , pueden representarse las curvas de 
supervivencia para los dos grupos de sujetos definidos por dicha variable y estudiar si son paralelas.

\section{Tipo de análisis}

Modelo Cox univariables: para cada variable que interviene en la ecuación se indica el coeficiente, su error estándar y el chi que permite calcular el nivel de probabilidad para ese coeficiente. (4) (Boj del Val, pág. 34).

\section{ETICA}

Esta investigación fue socializada para su validación y aprobación por el Comité de Ética del Hospital Regional de Occidente. Asimismo, se realizó previo consentimiento informado, respetando los principios de bienestar, justicia, equidad e igualdad; sin fines de lucro, con propósitos exclusivamente académicos, estrictamente confidencial; resaltando que la participación será voluntaria y anónima.

Los instrumentos que se utilizaron fueron validados, utilizando una prueba piloto de 25 pacientes seleccionados de la base central de datos de la unidad de endoscopía del Hospital Regional de Occidente, para determinar las variables de interés. Los pacientes seleccionados fueron de barrios, aldeas y colonias de Santa Rosa de Copán.

\section{RESULTADOS}

Realizando el análisis por el método de Cox para el estudio de la sobrevivencia, como resultados obtenemos los siguientes.

La sobrevivencia a 5 años después del diagnóstico de cáncer gástrico en la zona occidental de Honduras es del $14.29 \%$, dato que resulta inferior con relación al $20 \%$ propuesto en la hipótesis (ver tabla 1).

De los 22 casos que representan la sobrevivencia, 15 son del sexo masculino $(9.74 \%)$ y 7 del sexo femenino, equivalente al $4 \%$. 
Tabla 1. Sobrevivencia a 5 años del diagnóstico

\begin{tabular}{|l|l|l|l|l|l|l|}
\hline \multicolumn{2}{|c|}{ Número de casos de sobrevida después de 5 años } & \multicolumn{3}{c|}{ Total } \\
\cline { 1 - 4 } \multicolumn{1}{|c|}{ Pacientes } & Recuento & $\%$ & Recuento & $\%$ & Recuento & $\%$ \\
\hline & 98 & $63.63 \%$ & 34 & 22.07 & 132 & $85.71 \%$ \\
\hline Sin sobrevida & 98 & $9.74 \%$ & 7 & $4.55 \%$ & 22 & $14.29 \%$ \\
\hline Con Sobrevida & 15 & 73.37 & 41 & 26.62 & 154 & $100 \%$ \\
\hline$\%$ & 113 & \multicolumn{6}{|c|}{} \\
\hline
\end{tabular}

Fuente propia

En relación a la sobrevida por sexo, del total de la muestra 113 casos son del sexo masculino y sobreviven solo el $9.74 \%$ (15); 41 casos son del sexo femenino y sobreviven el $4.55 \%(7)$, esto demuestra que el sexo femenino tiene una sobrevida más alta por múltiples factores.

La tasa de sobrevivencia en meses en promedio, es de 26.82 después del diagnóstico; en mujeres es de 29.29 meses y en hombres de 25.93 meses. Ver Tabla 2.

\section{Tabla 2. Sobrevida posterior a 5 años}

\begin{tabular}{|c|c|c|}
\hline \multicolumn{2}{|c|}{ Numero de meses de sobrevida posterior a 5 años } & Total, promedio en meses \\
\hline Hombre & Mujer & \\
\hline 25,93 & 29,29 & 26.82 \\
\hline
\end{tabular}

Fuente propia

De los 22 casos, 16 sobreviven sin conocer qué tipo de tratamiento recibieron, 12 son hombres y 4 mujeres, el resto que son 6 pacientes, sí recibieron tratamiento. Se utilizaron como variables de tratamiento el quirúrgico, la quimioterapia y la radioterapia. De los 6 pacientes que sobrevivieron con alguno de estos tratamientos, 4 recibieron tratamiento quirúrgico (2 hombres y 2 mujeres), 3 recibieron quimioterapia (2 hombres y 1 mujer) y 1 mujer con radioterapia. Algunos recibieron combinación de tratamientos.

Tabla 3 Relación entre el tratamiento recibido y sexo de pacientes con sobrevida

\begin{tabular}{|l|c|c|}
\hline Tratamiento & \multicolumn{2}{|c|}{ Sexo del Encuestado } \\
\hline & Hombre & Mujer \\
\hline No se conoce si recibió tratamiento & 12 & 4 \\
\hline Tratamiento quirúrgico & 2 & 2 \\
\hline Tratamiento Quimioterapia & 2 & 1 \\
\hline Tratamiento Radioterapia & 0 & 1 \\
\hline
\end{tabular}

Fuente propia 
En cuanto a la variable distancia recorrida a los servicios de salud (acceso a Cesamo, Hospital de Área, Hospital Regional, Hospital Nacional y Atención Privada) del total (154) de los pacientes diagnosticados con Cáncer Gástrico se observa que el $31 \%$ de los pacientes no tienen accesibilidad oportuna (mayor de 2 horas), el $30 \%$ tienen una accesibilidad oportuna a los servicios de salud (menos de $30 \mathrm{~min}$ ), y el $36 \%$ con una accesibilidad intermedia (30 min-2 horas).

Tabla 4. Relación entre las variables distancia recorrida a los servicios de salud vrs tiempo

\begin{tabular}{|l|c|c|}
\hline $\begin{array}{l}\text { Distancia Recorrida } \\
\text { al centro de atención }\end{array}$ & Recuento & Porcentaje \\
\hline Menos de 30 Minutos & 46 & 30 \\
\hline Menos de 1 hora & 27 & 18 \\
\hline De 1 a 2 horas & 27 & 18 \\
\hline Mayor de 2 horas & 48 & 31 \\
\hline Sin respuesta & 6 & 4 \\
\hline total & 154 & $100 \%$ \\
\hline
\end{tabular}

Fuente propia

De los 154 casos en estudio, se observa que 63 (40.9\%) pacientes recibieron algún tipo de tratamiento ó tratamiento combinado.

Al relacionar la variable tratamiento recibido con la distancia recorrida, se observa que el tratamiento quirúrgico es el más accesible para el paciente; en los tratamientos, quimioterapia y radioterapia la accesibilidad es un factor limitante, debido que en la zona de occidente no se cuenta con estos servicios teniendo que realizar un recorrido mayor de dos horas, esto sin considerar el factor económico. 
Tabla 5. Relación entre tratamiento recibido y la distancia recorrida al centro de atención

\begin{tabular}{|l|c|c|c|c|}
\hline $\begin{array}{c}\text { Tratamiento } \\
\text { recibido }\end{array}$ & \multicolumn{4}{|c|}{ Distancia recorrida al centro de atención } \\
\hline $\begin{array}{c}\text { Menos de 30 } \\
\text { Minutos }\end{array}$ & $\begin{array}{c}\text { Menos de 1 } \\
\text { hora }\end{array}$ & $\begin{array}{c}\text { De 1 a 2 } \\
\text { horas }\end{array}$ & $\begin{array}{c}\text { Mayor de 2 } \\
\text { horas }\end{array}$ \\
\hline Quirúrgico & 16 & 3 & 2 & 10 \\
\hline Quimioterapia & 6 & 1 & 6 & 8 \\
\hline Radioterapia & 3 & 1 & 0 & 7 \\
\hline
\end{tabular}

Fuente propia

Al relacionar variable sexo y edad del paciente, de la población en estudio, (154 casos) se observa que la edad predominante en el diagnóstico de cáncer gástrico son los pacientes entre 55 y 64 años en ambos sexos, con una mayor incidencia en el sexo masculino.

En las personas menores de 40 años la incidencia es baja para ambos sexos.

Tabla 6. Relación entre la variable sexo y la edad en la población en estudio

\begin{tabular}{|c|c|c|}
\hline Rango de Edad & Hombre & Mujer \\
\hline Menores de 45 & 12 & 3 \\
\hline Entre 45 y 54 & 19 & 8 \\
\hline Entre 55 y 64 & 30 & 13 \\
\hline Entre 65 y 74 & 26 & 6 \\
\hline Mayores de 75 & 26 & 11 \\
\hline Total & 113 & 41 \\
\hline
\end{tabular}

Fuente propia

De los 22 casos que sobreviven a 5 años del diagnóstico, la edad de sobrevida oscila entre 55 y 64 años siendo mayor en el sexo masculino.

Esta tabla no evidencia sobrevida en pacientes menores de 45 años, Se podría considerar que los tipos de cáncer en personas jóvenes tienen un comportamiento más agresivo. 
Tabla 7. Relación entre la variable sexo y la edad en pacientes con sobrevida a 5 años

\begin{tabular}{|c|c|c|}
\hline Edad del paciente & Hombre & Mujer \\
\hline Menores de 45 & 12 & 3 \\
\hline Entre 45 y 54 & 19 & 8 \\
\hline Entre 55 y 64 & 30 & 13 \\
\hline Entre 65 y 74 & 26 & 6 \\
\hline Mayores de 75 & 26 & 11 \\
\hline Total & 113 & 41 \\
\hline
\end{tabular}

Fuente propia

El $100 \%$ de los pacientes fueron atendidos y diagnosticados en el Hospital Regional de Occidente; de estos, 117 (76 \%) recibieron atenciones posteriores al diagnóstico en el mismo hospital, 25 pacientes (16.4\%) fueron atendidos en el centro de salud más cercano, indicando que esta unidad de servicio tiene el mejor acceso para el paciente a los servicios de salud; 9 pacientes $(5.9 \%)$ se trasladaron a un Hospital Nacional, entendiéndose como nacional, el Hospital Mario Catarino Rivas y el Hospital Escuela Universitario.

22 pacientes recibieron atención en centros privados, lo que corresponde al $14.3 \%$

\section{Tabla 8. Atención médica recibida en la población en estudio}

\begin{tabular}{|c|c|c|}
\hline \multicolumn{2}{|c|}{ Recibió atención médica } & $\%$ \\
\hline Centro de Salud & 23 & 12 \\
\hline Hospital de Área & 18 & 9 \\
\hline Hospital Regional & 127 & 66 \\
\hline Hospital Nacional & 9 & 5 \\
\hline Atención Privada & 16 & 8 \\
\hline
\end{tabular}

Fuente propia

Del total de los 154 pacientes en estudio, 37 pacientes rechazaron el tratamiento médico, que corresponden al $24 \%$.

Entre las causas del rechazo del tratamiento las más significativas son la situación económica (62\%) causa predominante en ambos sexos y alcoholismo (16\%) factor predominante en el sexo masculino. 
Esto nos permite confirmar los datos de la tabla anterior (tabla 9) que indica que los pacientes de la zona occidental no tienen posibilidad de recibir el tratamiento pertinente a su diagnóstico en otros hospitales del país debido al factor económico.

Además de la necesidad de crear en el Hospital Regional de Occidente una unidad de tratamiento específico para esta patología, para ofertar otros tipos de tratamiento como ser quimioterapia y radioterapia diferente al quirúrgico que es el único ofertado actualmente; con el objetivo que la sobrevida de los pacientes diagnosticados sea mayor a los que refleja este estudio.

\section{Tabla 9. Relación entre las variables causas del rechazo al tratamiento médico según población en estudio}

\begin{tabular}{|c|c|c|c|c|}
\hline Causas de rechazo al tratamiento medico & hombre & mujer & total & porcentaje \\
\hline Situación Económica & 18 & 5 & 23 & $62 \%$ \\
\hline Alcoholismo & 5 & 1 & 6 & $16 \%$ \\
\hline Tratamiento Paliativo & 3 & 0 & 3 & $8 \%$ \\
\hline Desistió el tratamiento & 2 & 0 & 2 & $5 \%$ \\
\hline Etapa Terminal & 2 & 0 & 2 & $5 \%$ \\
\hline Otros & 1 & 0 & 1 & $3 \%$ \\
\hline
\end{tabular}

Fuente propia

El cálculo de los indicadores de pobreza (NBI) de los pacientes en estudio, según necesidades básicas insatisfechas (paredes, agua, excretas, piso, luz eléctrica y escolaridad); en el tiempo que padeció la enfermedad refleja: que de los 154 casos, el 61\% (94 pacientes) se encuentran en el estrato de pobreza, el 25.4\% (39 pacientes) en el estrato extrema pobreza, y 13.6\% (21 pacientes) en el estrato no pobre, esto confirma que el factor económico sigue siendo una variable determinante en la evolución de la enfermedad.

De acuerdo a la clasificación histológica de Lauren encontrada en el total de la muestra, el $41 \%$ (63) se encontraba en el estadio difuso, 42\% (65) en el estadio intestinal y $27 \%$ (19) en el estadio indeterminado, mixto $2 \%$ (3), NA $3 \%$ (4).

No es posible establecer una relación de sobrevida con la clasificación histológica, ya que no se cuenta con todos los criterios de clasificación por estadiaje TNM (método de estadiaje de neoplasias desarrollado por la AJCC (American Joint Committee on Cancer) en colaboración con la UICC (Union Internacional Contra Cancer). 
Tabla 10. Clasificación histológica del total de la muestra

\begin{tabular}{|c|c|c|c|c|}
\hline $\begin{array}{c}\text { Clasificación histológica } \\
\text { de Laurent }\end{array}$ & \multicolumn{4}{|c|}{ Total de la muestra } \\
\hline sobreviven & $\begin{array}{c}\text { Si } \\
\text { sobreviven }\end{array}$ & $\begin{array}{c}\% \text { de } \\
\text { sobrevida }\end{array}$ & $\begin{array}{c}\% \text { en base al total } \\
\text { de los casos }\end{array}$ \\
\hline Difuso & 54 & 9 & 41 & 6 \\
\hline Intestinal & 58 & 7 & 32 & 5 \\
\hline Indeterminado & 13 & 6 & 27 & 4 \\
\hline Mixtos & 3 & 0 & 0.0 & 0 \\
\hline NA & 4 & 0 & 0.0 & 0 \\
\hline total & 132 & 22 & $100 \%$ & 15 \\
\hline
\end{tabular}

Fuente propia

Por diagnostico endoscópico, la clasificación de Borrmann es de $66 \%$ en el estadío Tipo III siendo el más frecuente encontrado, confirmando que el diagnóstico es detectado de forma tardía para la efectividad del tratamiento.

La clasificación Borrmann Tipo I y II no son frecuentes en el diagnóstico, siendo esta una oportunidad de tratamiento más temprana, pero que no se logra diagnosticar a tiempo, esto tampoco es una oportunidad de sobrevida mayor porque es un diagnóstico para etapas avanzadas.

\section{Tabla 11. Clasificación macroscópica de Borrmann del total de la muestra}

\begin{tabular}{|c|c|c|c|c|}
\hline & \multicolumn{4}{|c|}{ Total de la muestra } \\
\hline $\begin{array}{c}\text { Clasificación } \\
\text { Borrmann }\end{array}$ & $\begin{array}{c}\text { No } \\
\text { sobreviven } \\
\text { Sobreviven }\end{array}$ & $\begin{array}{c}\text { Si de la } \\
\text { muestra }\end{array}$ & $\begin{array}{c}\text { \% en base al total } \\
\text { de los casos }\end{array}$ \\
\hline Tipo I & 0 & 0 & 0 & 0 \\
\hline Tipo II & 8 & 1 & 6 & 1 \\
\hline Tipo III & 87 & 15 & 66 & 10 \\
\hline Tipo IV & 11 & 2 & 8.4 & 1 \\
\hline Tipo V & 19 & 3 & 14 & 2 \\
\hline NA & 7 & 1 & 5.1 & 1 \\
\hline Total & 132 & 22 & $100 \%$ & 14 \\
\hline
\end{tabular}

Fuente propia 
La Tabla 12 Relación entre las variables edad y clasificación de Lauren refleja que los diagnósticos más frecuentes fueron la etapa difusa e intestinal en pacientes entre 45 a 64 años.

Tabla 12. Relación entre las variables edad y clasificación de Lauren

\begin{tabular}{|c|c|c|c|c|c|}
\hline & $\begin{array}{c}\text { Menores de } \\
45\end{array}$ & Entre 45 y 54 & Entre 55 y 64 & Entre 65 y 74 & $\begin{array}{c}\text { Mayores } \\
\text { de } 75\end{array}$ \\
\hline Difuso & 9 & 17 & 18 & 10 & 9 \\
\hline Intestinal & 3 & 8 & 20 & 16 & 18 \\
\hline Indeterminado & 1 & 2 & 4 & 5 & 7 \\
\hline Mixtos & 1 & 0 & 0 & 0 & 2 \\
\hline NA & 1 & 0 & 1 & 1 & 1 \\
\hline
\end{tabular}

Fuente propia

La Tabla 13 Relación entre las variables edad y clasificación de Borrman refleja que los diagnósticos más frecuentes fue Borrman tipo III en pacientes entre 45 a 64 años

Tabla 13. Relación entre las variables edad y clasificación de Borrman

\begin{tabular}{|c|c|c|c|c|c|}
\hline & $\begin{array}{c}\text { Menores de } \\
45\end{array}$ & Entre 45 y 54 & Entre 55 y 64 & Entre 65 y 74 & $\begin{array}{c}\text { Mayores de } \\
75\end{array}$ \\
\hline Tipo I & 0 & 0 & 0 & 0 & 0 \\
\hline Tipo II & 0 & 3 & 3 & 3 & 0 \\
\hline Tipo III & 12 & 18 & 29 & 18 & 25 \\
\hline Tipo IV & 1 & 3 & 4 & 1 & 4 \\
\hline Tipo V & 2 & 2 & 4 & 7 & 7 \\
\hline NA & 0 & 1 & 3 & 3 & 1 \\
\hline
\end{tabular}

Fuente propia 


\section{CONCLUSIONES}

1. La sobrevivencia a 5 años de pacientes diagnosticados con cáncer gástrico en los departamentos del occidente del país es del $14.29 \%$, cifra alarmante que indica que las expectativas de vida son muy limitadas debido a un diagnóstico tardío de la enfermedad y a las condiciones actuales de los sistemas de salud nacional que careen de una completa capacidad de respuesta, además de la disponibilidad que los gobiernos locales tengan en crear programas específicos para la prevención y control de esta patología.

2. Los factores que afectan la tasa de sobrevida son: edad (los hombres mayores de 60 años son las más afectados), sexo (se observa mayor sobrevida en la mujer porque acuden con mayor frecuencia a las unidades de salud), otros factores conocimiento del diagnóstico y estadio de la enfermedad, distancia hacia las unidades de salud y las necesidades básicas insatisfechas.

3. Del total de la muestra el porcentaje de paciente que no recibieron algún tipo de tratamiento específico para el cáncer fue de $68.2 \%$ (105) y el $31.8 \%$ (49) si recibió tratamiento.

4. De los 22 casos que sobrevivieron a 5 años del diagnóstico, solo 6 pacientes recibieron tratamiento específico (cirugía, quimioterapia, radioterapia) el resto recibió tratamiento de sostén de acuerdo a la capacidad de respuesta de la institución y al estadío de la enfermedad en el paciente.

5. Las relaciones entre las variables que influyen en la tasa de sobrevivencia de los pacientes diagnosticados con cáncer gástrico son: edad, sexo, fecha del diagnóstico y duración de la enfermedad, estadío vrs tratamiento, sobrevivencia vrs tipo de tratamiento, acceso a los servicios de salud vrs sexo, distancia recorrida vrs tratamiento, indicadores de pobreza vrs diagnóstico y tratamiento.

6. Causas por las cuales los pacientes diagnosticados con cáncer gástrico no recibieron tratamiento son: situación económica, alcoholismo, tratamiento paliativo, desistió el tratamiento, etapa terminal.

7. Dentro de las necesidades básicas insatisfechas de los pacientes estudiados el mayor porcentaje se encuentra ubicado en los estratos de pobreza y extrema pobreza. 
8. La tasa de sobrevivencia del total de la muestra de los pacientes que recibieron tratamiento quirúrgico es de $2.6 \%$, tratamiento de quimioterapia es de $1.9 \%$, tratamiento radioterapia $0.6 \%$. De los 22 casos que sobrevivieron a 5 años del diagnóstico el $18.2 \%$ recibió tratamiento quirúrgico, el13.6\% recibió quimioterapia y el $4.5 \%$ recibió radioterapia.

9. La tasa de sobrevivencia por estadiaje no puede realizarse ya que no se cuenta en el diagnóstico con los parámetros de clasificación internacional por estadiaje TNM. (Método de estadiaje de neoplasias desarrollado por la AJCC (American Joint Committee on Cancer) en colaboración con la UICC (Union Internacional Contra Cancer).

De acuerdo a la clasificación histológica de Lauren encontrada en el total de la muestra, el $41 \%$ (63) se encontraba en el estadio difuso, $42 \%$ (65) en el estadio intestinal y $27 \%$ (19) en el estadio indeterminado, mixto $2 \%$ (3), NA $3 \%$ (4).

Para la clasificación de Borrmann en el estadío Tipo III es el más frecuente encontrado $(68 \%)$, confirmando que el diagnóstico es detectado de forma tardía para la efectividad del tratamiento.

\section{RECOMENDACIONES}

1. Con los resultados obtenidos de este estudio, se recomienda replicarlo en el resto del país para lograr que a nivel de la Secretaria de Salud se cuente con una base de datos completa de todos los departamentos de la situación real de esta patología.

2. Con los datos obtenidos se sugiere a nivel de Salud Pública proponer estrategias para una política de salud que permita mejorar la calidad de vida de los pacientes y familias afectadas, de esta forma se estaría garantizando el derecho a la salud de la población hondureña.

3. Darle seguimiento al paciente diagnosticado y a sus familiares para proporcionar cuidados oportunos y adecuados a nivel primario y hospitalario.

4. Se recomienda proponer a nivel del gobierno central y en cada departamento de la zona de occidente, una campaña de promoción y prevención de estilos de vida 
saludable con el objeto de disminuir el número de casos registrados a través de diferentes medios de comunicación involucrando los diferentes actores de la sociedad civil.

5. Se recomienda optimizar el sistema de registro de la base de datos de la unidad de endoscopía del Hospital Regional de Occidente, para evitar sesgos en investigaciones posteriores; además de crear un sistema de registro a nivel nacional.

6. Se recomienda realizar una revisión de la base de datos proporcionada al cooperante posterior a este estudio, verificar datos nuevamente con los pacientes para rectificar resultados de esta investigación, debido a que este es un primer estudio sobre este tema, el tiempo limitado e inconvenientes no permitió la revisión de algunos datos importantes que pueden ser significativos.

\section{AGRADECIMIENTOS}

Se agradece a la Unidad de Endoscopia, al Departamento de Registros Médicos, Sala de Medicina de Hombres y de Medicina de Mujeres del Hospital Región de Occidente. Igualmente, a la Iniciativa del Occidente de Honduras para el estudio y prevención de cáncer gástrico. También se agradece al Registro Nacional de las Personas de los departamentos de Copán, Ocotepeque y Lempira; a los estudiantes de la Carrera de Enfermería y de las asignaturas de Biología y Química del CUROC. Gracias a la Coordinación Regional de Investigación del Centro Universitario Regional de Occidente y al director del Centro Universitario Regional de Occidente por su apoyo incondicional. Finalmente, a la Dirección de Investigación Científica y Posgrado de la UNAH por el apoyo logístico y financiero. 


\section{BIBLIOGRAFÍA}

Cárdenas, Rosario (2000). El uso de la autopsia verbal en el análisis de la salud. Estudios Demográficos y Urbanos. (665-683).Recuperado de: http://www.redalyc.org/articulo. oa?id=31204506

Chan A, Wong B, Feldman M, Grover S. (2013). Epidemiology of gastric cáncer. Recuperado de: https://www.uptodate.com/contents/epidemiology-of-gastriccancer

Eva Boj del Val. (2015) El Modelo de regresión de cox. Departamento de Matemática Económica, Financiera y Actuarial Facultad de Economía y Empresa Universidad de Barcelona (1-49).Recuperado de:http://diposit.ub.edu/dspace/bitstream $/ 2445 / 49070 / 11 /$ El $\% 20$ modelo $\% 20$ de $\% 20$ Cox $\% 20$ de $\% 20$ riesgos $\% 20$ proporcionales.pdf

Ferlay J. ShinHR. Bray F. Forman D. MathersC. Et al (2010) GLOBOCAN 2008 .Cancer incidence and Mortality Worlwide: IARC Cancer Base N. 10. International Agency for research on cancer, Lyon, France. Recuperado de: http://globocan.iarc.fr/Default.aspx

Ferlay J. ShinHR. Bray F. Forman D. MathersC. Parkin DM (2010) Estimates of worldwide burden of cancer in 2008: GLOBOCAN. Int J Cancer 127(12): 2893-2917, doi: 10.1002/ije.255

Universidad del Pais Vasco (2014). Indicadores de pobreza: diccionario de acción humanitario y cooperación al desarrollo. Recuperado de: http://www.dicc.hegoa.ehu.es/security/login.

Jemal A. Bray F. Center MM. Ferlay J. Ward E. Forman D (2011) Global cancer statistics. CA Cancer JClin61 (2):69-90.Doi:10.3322/caac.20107

Organización Mundial de la Salud (2004) Más allá de las cifras. Ginebra. 